\title{
Desarrollo de la práctica preprofesional en el contexto no presencial: experiencias en educación inicial
}

\section{Development of pre-professional practice in the non-classroom context: experiences in early childhood education}

\author{
Jessica Natalí Gallardo Ramírez \\ Universidad Católica Santo Toribio de Mogrovejo, Chiclayo, Perú \\ ORCID: https://orcid.org/0000-0002-2447-5591 \\ Silvia Georgina Aguinaga Doig \\ Universidad Católica Santo Toribio de Mogrovejo, Chiclayo, Perú \\ ORCID: https://orcid.org/0000-0001-6747-5375 \\ Osmer Campos-Ugaz \\ Universidad Católica Santo Toribio de Mogrovejo, Chiclayo, Perú \\ ORCID: http://orcid.org/0000-0002-3876-6605 \\ Ronald M. Hernandez* \\ Universidad Católica Santo Toribio de Mogrovejo, Chiclayo, Perú \\ ORCID: https://orcid.org/0000-0003-1263-2454
}

*Correspondencia:

Email: ronald.hernandez@outlook.com.pe
Citar como:

Gallardo, J., Aguinaga, S., Campos-Ugaz, O. \& Hernandez, R. (2021). Desarrollo de la práctica preprofesional en el contexto no presencial: experiencias en educación inicial. Propósitos y Representaciones, 9(3), e1444. http://dx.doi.org/10.20511/pyr2021.v9n3.1444 


\title{
Resumen
}

La investigación sistematizó el desarrollo de la práctica pedagógica en la carrera de educación inicial, durante la primera etapa de la pandemia Covid-19. En ese propósito, se realizó un estudio cualitativo, alineado a un diseño para el método sistematización de experiencias; reflexionándose sobre la evolución de la asignatura correspondiente, con la participación de 23 estudiantes, cada una a cargo de 20 niños y sus familias $(n=460)$, con el acompañamiento de la docente universitaria. Los resultados fueron; aplicación de estrategias didáctico-pedagógicas de los procesos en el contexto remoto; empleo de recursos digitales potenciados creando materiales contextualizados para la interacción y; el abordaje del acompañamiento emocional ineludible frente a la vulnerabilidad de la infancia. Al término del estudio se concluye que, es imposible desvincular la tecnología educacional, de los aspectos afectivos, que bien fueron asumidos traduciendo en logro de aprendizaje, los esfuerzos colectivos en trabajo consensuado de escuelas, familias y universidad.

Palabras clave: Práctica pedagógica; Pandemia; Tecnología educacional; Infancia.

\begin{abstract}
The research systematized the development of pedagogical practice in the initial education career, during the first stage of the Covid-19 pandemic. For this purpose, a qualitative study was carried out, aligned to a design for the systematization of experiences method; reflecting on the evolution of the corresponding subject, with the participation of 23 students, each in charge of 20 children and their families $(n=460)$, with the guidance of the university professor. The results were; application of didactic-pedagogical strategies of the processes in the remote context; use of enhanced digital resources creating contextualized materials for interaction and; the approach to the unavoidable emotional accompaniment in the face of childhood vulnerability. At the end of the study, it is concluded that it is impossible to unlink educational technology from affective aspects, which were well assumed, translating into learning achievement, collective efforts in consensual work of schools, families and universities.
\end{abstract}

Keywords: Pedagogical practice; Pandemic; Educational technology; Childhood.

\section{Introducción}

El año 2020 en el mundo, se constituyó como uno de los más difíciles de afrontar debido a la pandemia por COVID-19, remeciendo los ámbitos económico, político, social y educativo (Trilla, 2020). Este último sistema, asumió el desafío de transformar los mecanismos pedagógicos para adaptarse a un contexto no presencial de trabajo remoto y asegurar la continuidad del servicio, sobre todo, la calidad educativa, a la luz de sin número de limitaciones (Araujo et al., 2020).

En esas circunstancias, el Banco Interamericano de Desarrollo (BID, 2020) y Universia Banco Santander convocaron a una reunión virtual, asistiendo rectores de universidades líderes de América Latina, quienes buscaban discutir cómo enfrentar desde las universidades los retos y desafíos producto de la pandemia. Los resultados del diálogo fueron, generar mecanismos de transformación digital que viabilicen rápidamente el proceso educativo superior universitario (Vicentini, 2020).

Siendo coherente con estas disposiciones previas, se pronunció el organismo adscrito al Ministerio de Educación, SUNEDU (Superintendencia Nacional de Educación Superior Universitaria), mediante resolución del consejo directivo $\mathrm{N}^{\circ}$ 039-2020-SUNEDU-CD, quienes autorizaron el desarrollo de las actividades universitarias vía online, motivando al pronto inicio de las labores académicas. Tomando en cuenta que las casas de estudio licenciadas han 
implementado previamente plataformas virtuales de aprendizaje, como requisito relacionado a estándares de calidad, esto sirvió como punto de partida y junto a otros mecanismos efectuados, constituyó una ventaja, concretándose el inicio de clases no presenciales en el nivel de educación superior. Sin embargo, la epidemia cambió la vida de 33 millones de peruanos, de los cuales se vieron afectados aproximadamente 174.000 estudiantes, quienes abandonaron los estudios por múltiples razones vinculadas a la coyuntura nacional (Alayo, 2020).

Mientras tanto, el sistema para la educación básica asumía diversas realidades. En efecto, desprovistos de plataformas educativas, en su gran mayoría, especialmente las instituciones educativas del sector estatal de todos los niveles sufrieron en diferentes proporciones los embates de la emergencia sanitaria. Según MINEDU (2021) más de 40 mil niños podrían haber dejado de atenderse si no hubieran recibido el apoyo de universidades públicas y privadas por medio de sus practicantes, considerando que los profesionales en formación cumplirían roles relevantes. Cabe resaltar entonces, la gran importancia del desarrollo de las prácticas preprofesionales que, bajo esquemas coyunturales se configuró como un proceso favorecedor en manos de jóvenes dispuestos a sumar de forma entusiasta, valiéndose de lo aprendido en los ciclos de formación universitaria.

De hecho, la pandemia dinamizó toda acción posible, de modo que, al inicio se superaron algunas comunes dificultades de acceso a los grupos escolares y el llamado practicante asumió un papel más significativo, luchando de manera conjunta con el profesorado, frente a los vacíos técnicos de la conectividad y los procesos mismos para consolidar las experiencias de aprendizaje utilizando herramientas tecnológicas y recursos digitales. En esa línea, destaca la aseveración acerca del manejo de las tecnologías que hicieran Llamas-Salguero y Gómez (2018), tratando las cualidades de una generación especial en la formación inicial docente, las mismas que permiten liderar de manera compartida las actividades escolares remotas, articulándose a los propósitos educacionales.

Ciertamente, esto implicó de forma antelada que desde las universidades se reestructurara la ejecución de la práctica, abordando no solo la atención a las necesidades de los estudiantes y docentes con el soporte de las TIC, sino que se debía mantener el sentido mismo de este espacio exploratorio, experimentador, rico en aprendizajes, en constante interacción con los actores educativos. Sin duda estas experiencias, nutren el acervo profesional de los futuros maestros y promueven la actualización de los planes curriculares en pregrado. En tal sentido, algunos elementos que argumentan la importancia de desarrollar las prácticas preprofesionales de manera online y sistematizarlas, se delinean en la idea anterior y su significado alimentador del currículo a nivel de las casas formadoras, sobre todo, si se considera la misión de las universidades como centros de producción de conocimiento. En síntesis, los estudios materializados en documentación producida y el impacto que generan, se filtran en escenarios del saber científico y la calidad educativa (Gonzalez-Diaz et al., 2022); más todavía, se trata de recuperar de contextos específicos y de la práctica, aquello que nutrirá la teoría y conocimiento actualizado.

A propósito, Mateus et al. (2022), hacen mención de los aprendizajes fruto de las prácticas y experiencias como clave para construir críticamente la agenda educativa en cada región y sugieren la realización de estudios como este que describe, analiza e interpreta las ocurrencias vividas por estudiantes universitarios durante la no presencialidad, apoyando a las instituciones educativas preescolares del estado en la ciudad de Chiclayo.

En tal sentido, el presente estudio esgrime las estrategias para el aprendizaje que han pasado por un proceso de transformación digital en el nivel de educación inicial, acercando a niños, profesores, padres de familia en el marco de la educación remota, razón por la que surge la premisa ¿cómo desarrollan la práctica preprofesional estudiantes de nivel inicial en el sistema no presencial? 
Cabe enfatizar, que las cuestiones metodológicas de la investigación giran en torno a la máxima aspiración, cual es, sistematizar el desarrollo de la práctica preprofesional en el contexto no presencial y en el nivel de educación inicial, justificándose en la importancia de recoger dificultades, aciertos y retos en el aprendizaje de los niños en preescolar. Del mismo modo, es una contribución para la difusión de estrategias y recursos empleados durante la emergencia sanitaria COVID-19, haciendo referencia al acompañamiento pedagógico, tecnológico y emocional. Entretanto, se identificaron como beneficiarios del aporte teórico-práctico, los docentes en formación y aquellos en ejercicio, dado que esta sistematización servirá como insumo para mejorar su quehacer pedagógico.

Siendo así, sistematizar la experiencia envuelve un proceso genuino retroalimentador de las acciones en las universidades y la formación inicial docente; en ello estriba el valor del estudio, acercándose de manera ideal a las reflexiones que fortalecen y encaminan hacia la mejora continua en cualquier contexto y todo campo disciplinar.

A propósito, en referencia a la retroalimentación formativa la que se dirige al logro de aprendizajes, esta se construye sólidamente a partir de un vínculo de confianza entre los actores de un proceso (Cabero et al., 2017). Sin duda, ahora se extiende la acción, incorporándose bajo metas compartidas, las desarrolladas desde la universidad, a través de la práctica preprofesional, con sentido unificador de esfuerzos junto a directivos, docentes, estudiantes y familias, intercambiando información cualitativa sobre logros, desafíos y los modos en que una producción puede ser mejorada.

Considerando que sean útiles estas acciones reflexivas, se necesita partir de planificar maniobras nuevas en escenario virtual, ciertas estrategias didácticas donde el educador promueve procedimientos para el aprendizaje significativo de los estudiantes y prioriza el cumplimiento de los objetivos (Flores, 2017; como se citó en Ruiz y Vásquez, 2019).

Sin embargo, otros mecanismos, se hacen imprescindibles, como los referidos a soporte virtual, presencia innegable que expande el nivel de alcance de los docentes comúnmente acostumbrados a los espacios áulicos exclusivos de la enseñanza aprendizaje y que terminan combinando lo pedagógico-curricular con lo tecnológico, tal como el modelo TPACK sugiere (Cabero et al., 2017).

Desde luego, y de acuerdo con Vialart (2020) y Diego-Mantecón et al. (2021), es conveniente no solo implementar entornos virtuales, sino que estos se orienten bajo esquemas de proyectos colaborativos que promuevan la construcción de conocimiento, asumiendo temáticas de vanguardia relativos a ciencia, tecnología, ingeniería y matemáticas (STEAM).

\section{Método}

El desarrollo del estudio cualitativo estuvo conformado por 23 estudiantes universitarias, cursando entre el V y X ciclo de la carrera educación inicial, en una Universidad privada de Chiclayo; quienes asumieron el acompañamiento de 20 familias respectivamente, haciendo un total de 460 participantes. En ese sentido, el recojo de la información diagnóstica fue posible haciendo uso de la lista de chequeo incluida en el protocolo de orientaciones pedagógicas dirigido a tutores de acogida, versión 2.0 (MINEDU, 2020).

El procedimiento se evidencia desde el planteamiento de algunas interrogantes (preguntas iniciales), posteriormente se origina el proceso de interpretación orientada a la comprensión profunda de las experiencias vividas (recuperación del proceso vivido), asumiendo que las reflexiones impactarán en la evolución de conocimientos teóricos anteriores, debido a eventos en el escenario de la praxis (reflexión de fondo), para finalmente formular conclusiones y comunicar lo aprendido (puntos de llegada). 
Analizar la información en la investigación es hacer uso del método sistematización de experiencias (Carvajal, 2018; Jara, 2020), el mismo que permite interpretar de forma crítica una o varias vivencias ordenando y reconstruyendo las mismas para explicar el proceso con sentido lógico, algunos factores intervinientes, las relaciones acontecidas y el sustento que justifica determinada práctica. Además, se contrastan con otras experiencias y la teoría existente, significando una contribución desde y para la práctica (Unday \& Valero, 2017; Schwartzman et al., 2021). En la Figura 1 se esboza el diseño de la metodología empleada, resaltando el uso de bases científicas suficientes (Teoría necesaria), para las comparaciones entre experiencias de otras realidades y a la luz de la teoría.

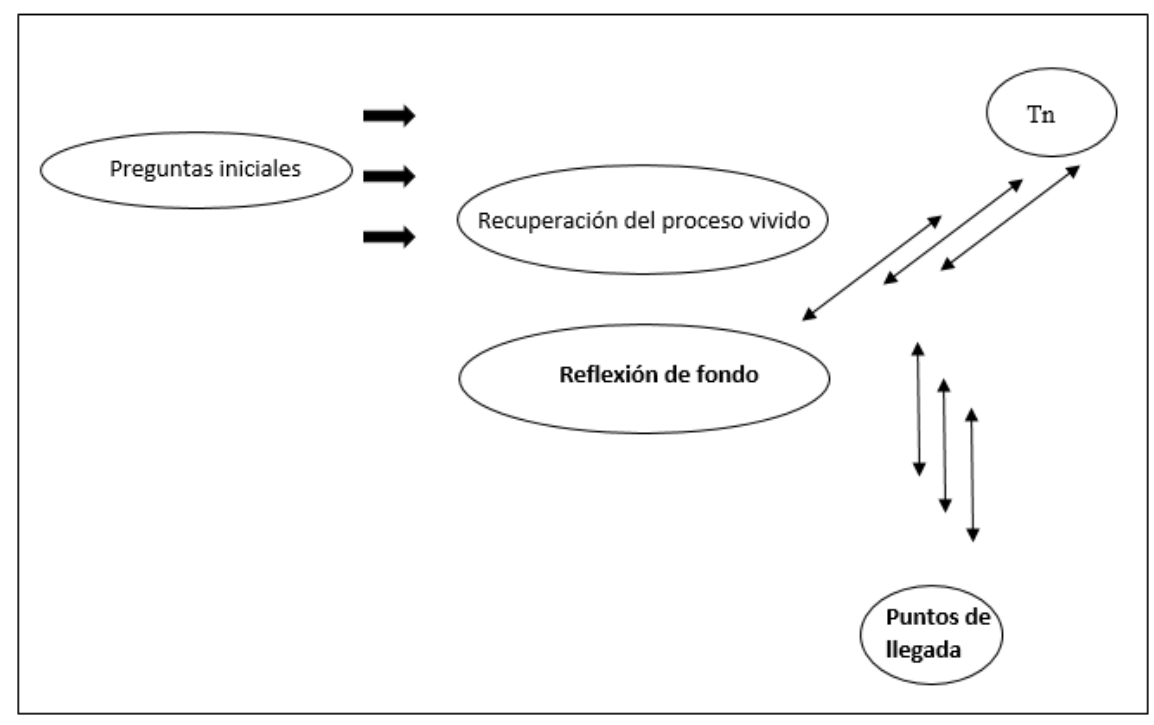

Figura 1. Modelo de sistematización de experiencias, tomando en cuenta la propuesta metodológica de Jara (2020)

\section{Resultados}

A fin de reinventar las formas de enseñar y aprender durante la pandemia, es importante pensar fuera de la caja "Think outside the box", esta frase se hace presente en el CADE (2016), evento organizado por IPAE que relaciona el aprendizaje en un mundo digital. La pandemia ha evidenciado la necesidad de meditar una propuesta en nuevas condiciones y oportunidades con un enfoque en el bienestar de toda la comunidad educativa y las diferentes formas de acompañamiento, asumiendo estrategias desde el empoderamiento. Sin embargo, también es fundamental reflexionar en relación a las estrategias no solo con el fin de lograr un propósito pedagógico, sino más bien como aquellas que ayuden a identificar cómo se logran estos y cual es aprendizaje adquirido en el proceso. Lo anterior implica, diseñar algo más que un conjunto de actividades respondiendo al problema auténtico (confinamiento por pandemia) y tomando en cuenta ampliar el repertorio de posibilidades pedagógicas, de la mano con lo tecnológico, pero incorporando también, aspectos emocionales para las buenas prácticas.

\section{Estrategias emergentes que incluyen el acompañamiento pedagógico.}

Los procesos pedagógicos inherentes a la práctica pedagógica, son abordados en el desarrollo de la sistematización, destacando que, en torno a las oportunidades de acceso remoto a las aulas, los estudiantes de la carrera de educación inicial, desempeñaron un papel muy valioso y, en trabajo colegiado desde la gestión entre las instituciones involucradas (universidad y jardines de infancia), se propiciaron experiencias enriquecedoras bajo una meta compartida, no solo mantener el servicio educativo, sino otorgar esperanzadoras prácticas en un momento crucial para la educación a nivel mundial. 


\section{Abordaje desde la planificación.}

La planificación anticipada fue una de las mejores estrategias, usando para tal fin, guiones de clase que orientarían el trabajo de inicio a fin, los días asignados para práctica. En un formato detallado de la acción educativa, cartillas informativas elaboradas en Canva, anticipaban a las familias la ruta a trabajar, contextualizado la estrategia nacional multicanal Aprendo en casa. Esta cartilla contenía links de acceso a sala Zoom y Google Drive de materiales a emplear, en los cuales se incluyeron tutoriales haciendo uso de vídeos. Así mismo la información era compartida desde de los canales de WhatsApp y/o grupos privados en Facebook, creados con los contactos telefónicos de las familias y los e-mails personales.

\section{Ejecución de las sesiones de aprendizaje remoto.}

Como parte de la práctica preprofesional, las estudiantes llevaron el desarrollo de clases no presenciales, valiéndose, principalmente, de estrategias del ABP (aprendizaje basado en proyectos). Esta iniciativa surge en la necesidad de realizar simulaciones con los menores a través de Zoom y/o Google Meet. Cabe señalar, que el uso de las plataformas de video conferencia constituía al inicio de la pandemia, un mecanismo bastante riesgoso y poco usado por el profesorado, de modo que sirvió de manera experimental para recoger exitosamente algunas respuestas como observar a los niños interactuar muy motivados, intercambios a tiempo real desde una pequeña pantalla, que permitieron escuchar y ser escuchados, visualizar y ser vistos mientras aprendían junto a sus familiares a respetar normas básicas para el encendido y apagado de micros y cámaras. Todo lo anterior, era el reflejo de una necesidad imperiosa de interacción. En este contexto resultó ventajoso realizar un acompañamiento pedagógico. De la cotidianidad, se rescataron elementos y recursos accesibles a ellos, desde sus casas, se pudieron ejecutar actividades de cocina o jardinería, por ejemplo, involucrando directamente a la familia bajo la guía de la maestra. De hecho, estas fortalecen el trabajo pedagógico y se convierten en actores coprotagónicos de los procesos, soportes principales que incluso contribuyeron en la creación de espacios físicos al interior de sus viviendas simulando las aulas de preescolar, rincones desde donde tomaban las sesiones virtuales, generando emocionalmente, fuerte vínculo con la comunidad educativa.

Entretanto la estrategia nacional Aprendo en casa propicia que el uso de medios audiovisuales, como cuentos y canciones creados por las estudiantes sean empleados con mayor frecuencia y que además se aborden dentro de la práctica creando contenido propio para ser compartido con los usuarios, convirtiéndose en apoyo permanente, asumiendo el rol de ayudantía a la docente de aula desde el trabajo no presencial.

\section{Fortalecimiento de los procesos de evaluación.}

Evaluar usando recursos digitales para acompañar el proceso pedagógico a las familias desde la práctica preprofesional ha constituido un reto, pues se requería sincerar horarios y la disposición; así mismo la conectividad a internet era una limitante. Según los resultados obtenidos con la aplicación de la lista de chequeo del protocolo ofrecido para tutores de acogida (MINEDU, 2020), la disposición de la comunicación de las familias durante el día fue así; el 23\% se conectaba en la noche, $36 \%$ en la mañana y $41 \%$ en la tarde. Entonces se decidió crear varios horarios para desarrollar la evaluación formativa basada en el proceso de retroalimentación. Sin duda, ello permite realizar devoluciones informando de manera descriptiva logros o progresos, los que se vinculan al nivel de competencia esperado; es decir, basados en criterios de claridad y compartidos al niño y su familia, ciertos esquemas de trabajo o mecanismos procedimentales permiten comparar cualitativamente "lo que debió hacer, lo que intentó lograr con lo que efectivamente hizo" (MINEDU 2017, p. 180). 
Las notas de campo creadas en el portafolio digital usando Google Docs 365, fue de gran ayuda, pues constituyó un insumo importante, haciendo posible la identificación de aciertos, errores recurrentes, así como aquellos aspectos que más atención requieren. A partir de ello y el diálogo con los niños y sus familias se brindaba información oportuna, promoviendo la reflexión y la búsqueda de estrategias que le permitan consolidar sus aprendizajes.

A propósito, de la experiencia en el desarrollo de la evaluación como proceso pedagógico, se destaca lo beneficioso que fue potenciar genuinamente el diálogo entre los actores, en este caso, incluyendo la participación activa de la practicante, sumando esfuerzos conjuntos. En síntesis, se dinamizaron espacios dialógicos compartiendo información valiosa bajo las formas de autorreflexión, donde las niñas y los niños experimentaban analizar fortalezas y debilidades, a partir de las cuales eran capaces, a pesar de su corta edad, de plantear mecanismos de mejora hacia el logro de sus aprendizajes. Durante este proceso de acompañamiento fue clave el apoyo del principal aliado, el familiar responsable, exigiendo por parte de las docentes en formación, desplegar capacidades de creatividad, comunicación, compromiso y responsabilidad.

Definitivamente, la etapa de evaluación formativa caracterizada por la retroalimentación es viable en base a desarrollarse en horarios consensuados y se aprovecharon al máximo los recursos tecnológicos con audios y videos para el acompañamiento asincrónico.

Los recursos digitales que son utilizados en el acompañamiento tecnológico a los niños y familias en el proceso de adaptación de la práctica preprofesional durante el trabajo remoto se hacen visibles en la aplicación del instrumento de recojo de información. El mecanismo evidenció que el uso de los recursos tecnológicos haciendo posible la conectividad para el desarrollo de las clases no presenciales van en un 18\% de familias que usan una computadora, $32 \%$ de familias emplean el celular, 32\% la televisión, 9\% telefonía, un $4 \%$ radio y 5\% otro tipo de canales tecnológicos; en ese sentido la atención a los niños se diversifica de acuerdo al canal de acceso empleado para conectarse, con estrategias para el adecuado acompañamiento tecnológico a las familias.

Cabe resaltar que una de las principales dificultades para acompañar a través de un celular o computadora fue la conexión a internet, la misma que suele presentar fallas constantes; sin embargo, constituye un reto para seleccionar aplicaciones que puedan ser usadas desde el celular, sin datos adicionales como las redes sociales Whatsapp y Facebook; incluso crear contenido digital de fácil acceso que pueda ser empleado desde un celular.

Por otro lado, se fortaleció el manejo y creación de material didáctico en herramientas digitales más conocidas como Word, Power Point y Paint, para la creación de afiches, secuencias, juegos, entre otros. También se emplearon hojas de cálculo Excel para registros de asistencia de los niños, cotejados a su vez, con los audios Whatsapp de cada niño, formalizando así la asistencia.

Del mismo modo, más aplicaciones continúan explorándose para la elaboración de material audiovisual, infografías o cartillas en Canva y Pixart, las que al inicio eran básicas y luego interactivas ocasionando mayor interés de los niños por seguirlas y mantener atención conjunta.

Entretanto, los editores de vídeo como Inshot y/o Filmora fueron aquellos que posibilitaron el empleo de tutoriales para la elaboración de los materiales y para interactuar con los niños empleando recursos literarios como cuentos y canciones creadas por las estudiantes. Asimismo, las aplicaciones Disney, permitieron personalizar el material digital, muy útil en ocasiones celebratorias como los cumpleaños; sin duda, el escuchar sus nombres y observar imágenes y fotografías propias insertadas en los videos, se ocasionaban efectos emocionales y afectivos importantes acercando indiscutiblemente a los miembros de la familia y la institución. 
Una de las herramientas más usadas para apoyar a las familias con el ordenamiento y archivo de los recursos empleados y las evidencias es el Google Drive; las estudiantes de práctica pre profesional se hicieron responsables de enseñar y acompañar a los padres quienes podían optimizar el uso de la memoria de sus dispositivos. Del mismo modo, usaban el drive para la organización y documentación de las evidencias y crearon notas de campo y agendas digitales empleando Trello.

Otra herramienta muy útil son los formularios Google, esta vez sirvieron en la circulación de instrumentos como cuestionarios a las familias, sea el sugerido por MINEDU para recoger información relacionada a la disposición familiar en el trabajo no presencial, situación laboral, conectividad, uso de equipos tecnológicos y condiciones médicas en tiempos de pandemia. Entre otros, este mecanismo fue empleado también para actividades de compresión de texto, insertando secuencia de imágenes y/o vídeos. De hecho, circularon también, pequeñas encuestas para conocer, por ejemplo, los gustos y preferencias de los niños y niñas.

Acerca de las plataformas Zoom, Meet y Google Classroom, estas habían sido poco o nada conocidas por los estudiantes, quienes aprendieron a usarlas y se convirtieron en las principales plataformas para el intercambio sincrónico y asincrónico. Una vez por semana, se propiciaban los encuentros con este soporte tecnológico, lográndose que tanto los niños como sus familias ejercitaran en sala virtual, las netiquetas; identificándolas como acuerdos consensuados en el mundo digital del que indefectiblemente ya son parte todos.

Con respecto a la creación de contenido digital, la grabación de vídeos se convirtió en un recurso muy común, sin embargo, acorde con los avances de la era digital, la reproducción y difusión de las producciones cobraron fuerza en plataformas de redes sociales y canales de YouTube y Facebook Live, favoreciendo la transmisión en vivo y en diferido para las familias que no podían acceder por dificultades de conectividad u otro tipo.

El buscar diversas opciones de innovar en la práctica generó la dinámica espiral que concreta la exploración en aplicaciones poco conocidas hasta el momento: Educaplay, Quizizz, Kahoot o Socrative Student, con ellas se inició la creación de juegos, dinámicas, entre otros, logrando diversas formas creativas de aprender. Incluso, aplicaciones como Power Point sirvieron en la creación de contenidos digitales para promover la participación activa de los niños y sus familias, generando emoción y manteniendo fuerte vínculo con el proceso formativo. Asimismo, se articularon las temáticas de Aprendo en casa, logrando que juegos de ruletas, memoria y rompecabezas sean los favoritos de los niños. Cabe enfatizar que, las aplicaciones elegidas y materiales creados fueron pensadas en que las familias puedan acceder a ellas desde su celular y además no tengan que descargarse, sino más bien, a través de un enlace URL, esté disponible en línea con la finalidad de no congestionar la memoria del dispositivo más comúnmente usado para las clases remotas.

Retos y desafíos abordados desde el acompañamiento emocional a niños y familias durante el proceso de adaptación al trabajo remoto.

Tener mirada empática, amable, reconocimiento del otro, no invalidar alternativas planteadas por las familias, desprejuiciada, creativa y pensar de manera colaborativa cómo podemos aprender desde casa, eran los principales planteamientos que viabilizaron el acompañamiento emocional en tiempos de incertidumbre. Cabe destacar que utilizar un lenguaje amable generador de confianza fue determinante, así como ser tolerante y empático frente a múltiples situaciones producto de fallas técnicas que interrumpían las interacciones virtuales; se asumieron entonces, como parte del proceso de aprender sobre la marcha a usar los recursos y herramientas tecnológicas. 
Al referirse a la pandemia por COVID-19, es ineludible mencionar la pérdida de vidas humanas afectando a muchos hogares, situación no ajena al entorno de la práctica pedagógica. Asimismo, estos eventos sui generis exigieron la adaptación al escenario no presencial y los niños pasaban largas horas frente al televisor/computadora para escuchar el programa Aprendo en casa. La estrategia por sí misma carecía de receptividad ocasionando rechazo a las actividades educativas y en atmósferas de tensión, niños llorosos y desmotivados era una situación común. En estas circunstancias, los estudiantes universitarios asumieron un papel de acompañamiento brindando soporte a niños, niñas y sus familias afectadas con el virus. Esto implicó, una comprometida labor desde el buen trato y la pronta creación de estrategias hacia la incorporación activa de los menores en las acciones educativas. Así, junto a sucesivos intentos y una creciente gamma de recursos didácticos y tecnológicos empleados empezaron a manifestarse cambios en la receptividad tanto de los niños, como de sus familiares más próximos, terminando involucrados activamente en el logro de aprendizajes.

Entre las principales acciones concernientes al aspecto emocional, la reflexión guiada en torno a la promoción de la autonomía, el autoconcepto, respeto de sus ideas y participación activa en la toma de decisiones para la organización de su actuar escolar; favoreció disminuir actitudes de interferencia por parte de los padres en la actividades y sesiones en las plataformas de video conferencia.

Algunas estrategias para el acompañamiento emocional fue usar la musicoterapia y el mindfulness, activaciones desarrolladas en las plataformas de uso sincrónico, junto a ejercicios de respiración; favoreciendo sin duda el equilibrio y regulación emocional.

Por último, es importante mantener la comunicación asertiva y ejercitar la empatía, esto permitió hacer efectivas las interacciones entre niños y sus familias, estudiante universitaria y docente de aula, generando una mejor comunicación en medio de situaciones adversas afectando a todos.

\section{Discusión}

La Red Kipus en la publicación de la literatura que recopila el diálogo y reflexiones del último encuentro de experiencias para la formación en estos tiempos, en el cual participaron los representantes de las instituciones de educación superior, pone de manifiesto que el reto ha sido complejo, porque se ha requerido de un proceso de adaptación que pasa de una modalidad presencial a una muy distinta para llevar a cabo las prácticas preprofesionales. Asimismo, se ha requerido incorporar el dominio de competencias digitales que hagan posible el acompañamiento a los niños en EBR (Blanco et al., 2021), la familiarización de las plataformas digitales y en ese sentido, el rediseño de las actividades educativas, todo esto articulado a la estrategia Aprendo en casa propuesta por el MINEDU

Así, las estrategias emergentes en esta coyuntura de pandemia por la COVID-19 que ha posibilitado el acompañamiento pedagógico durante el proceso de enseñanza aprendizaje y evaluación, de las estudiantes universitarias en la carrera educación inicial, se ligaron exitosamente a la planificación de actividades pedagógicas y procesos evaluativos formativos considerando el contexto excepcional. Se enfatizó en la retroalimentación a partir de la presentación de productos o evidencias de aprendizaje, a través de canales de comunicación digital o aplicaciones en Google; procesos en constante reflexión junto a las docentes de aula y familias, en favor de los niños y niñas.

En concordancia con lo expresado Valencia (2020) señala que las oportunidades de aprendizaje ofrecidas desde la no presencialidad promueven en el universitario un rol más decisivo y partícipe del proceso de retroalimentación, reflejándose estas acciones en la práctica pedagógica con los niños de tal forma que se fortalecen acciones educativas tomando como parte 
medular de todo proceso evaluador, aquello que permite tomar decisiones para replantear o reforzar aprendizajes.

Hernández-Palma et al., (2020), sostiene que las decisiones vinculadas con los recursos tecnológicos estuvieron orientadas a la elección de plataformas, herramientas y aplicaciones versátiles y amigables, que pudieran ser utilizadas por los estudiantes desde cualquier equipo electrónico: computadoras, tabletas y teléfonos. La versatilidad ha sido un requisito para disminuir la brecha de acceso y de continuidad del proceso formativo de los estudiantes. Es por ello que, uno de los recursos más utilizados en la interacción docente-estudiante ha sido el WhatsApp. No obstante, se ha reconocido que, a pesar de los esfuerzos tanto de las instituciones formadoras y de los propios estudiantes, existen problemas de acceso a equipos y de conectividad.

También, Valencia (2020) precisa sobre riesgos asumidos al desarrollar las experiencias de aprendizaje de manera remota, la promoción del individualismo y el aislamiento, situación que no se llegó a evidenciar en la facultad de ciencias de la educación de la Universidad Católica del Oriente, pues las TIC ofrecen diversas formas de interactuar de manera auténtica de forma síncrona y asíncrona. Además, las comunidades de aprendizaje de docentes y estudiantes acreditan mucha riqueza en el número y la calidad de las interacciones, este proceso formativo en los practicantes ha generado reflexiones de manera que, en el desarrollo de la práctica se incorpore el uso del ciberespacio, las tecnologías y los diversos medios de comunicación.

En resumen y concordancia con el citado autor, constituye un reto y desafío, destacar la práctica desde un proceso reflexivo que reconfigure la experiencia pedagógica y fortalezca los lazos de cooperación mutua, generando sostenimiento y contención emocional a las familias, sobre todo a los más pequeños, priorizando la gestión de las emociones en una situación adversa y de dificultad como la creada por la pandemia por Covid-19.

\section{Conclusiones}

Los docentes en formación quienes bajo el rol de mediador asumieron el $50 \%$ de responsabilidad en el proceso formativo de los niños y las familias como aliados el otro $50 \%$, han demostrado que es posible desarrollar verdaderas coaliciones de aprendizaje y amor en favor de la educación peruana.

Las experiencias de aprendizaje compartidas contribuyen al diseño de estrategias para desarrollar competencias en contextos desafiantes y disruptivos, siendo fundamental la buena disposición y el adecuado aprovechamiento de las TIC en favor de la pedagogía; desarrollando además, capacidad crítica y el planteamiento de hipótesis entre los infantes, introduciendo de esta manera las áreas STEAM, desde plataformas de video conferencia y el aprendizaje basado en proyectos.

El soporte emocional gestionado por las estudiantes universitarias es determinante en el acompañamiento que brinde a las familias, siendo la clave, enfatizar en la formación en coaching para el empoderamiento y uso de estrategias en contextos diversos.

El estudio deja como lección aprendida que, son imprescindibles las actividades de contención emocional como sesiones de relajación y el involucramiento de las familias durante el proceso formativo de los niños en el nivel inicial.

La investigación promueve alianzas entre la experiencia del docente en ejercicio profesional y las fortalezas de jóvenes generaciones de maestros cercanamente vinculados con la tecnología, expandiendo la oportunidad de renovar la propuesta pedagógica y valorar el rol del docente como facilitador y gestor del aprendizaje. 


\section{Referencias}

Alayo, F. (2020). Unos 174.000 estudiantes peruanos dejaron la universidad en lo que va del 2020. Diario el Comercio. https://elcomercio.pe/lima/sucesos/unos-174000-estudiantesperuanos-dejaron-la-universidad-en-lo-que-va-del-2020-noticia/

Araujo, L. E., Ochoa Mogrovejo, J. F. \& Vélez Verdugo, C. (2020). El claroscuro de la universidad ecuatoriana: los desafíos en contextos de la pandemia de COVID-19. Revista Digital de Investigación en Docencia Universitaria, 14(2). http://dx.doi.org/10.19083/ridu.2020.1229

Banco Interamericano de desarrollo. BID (2020) La Educación superior en tiempos de Covid-19. https://publications.iadb.org/es/publications/spanish/document/La-educacion-superioren-tiempos-de-COVID-19-Aportes-de-la-Segunda-Reunion-del-Di\%C3\%A1logoVirtual-con-ectores-de-Universidades-Lideres-de-America-Latina.pdf

Blanco, M. A., Blanco, M. E. \& Hinojo, B. T. V. (2021). Actividades de bienestar emocional propuesta para el desarrollo del aprendizaje significativo en tiempos de postpandemia. Revista Conrado, 17(80), 330-338. https://conrado.ucf.edu.cu/index.php/conrado/article/download/1851/1817

Cabero, J., Roig, R. \& Mengual, S. (2017). Conocimientos tecnológicos, pedagógicos y disciplinares de los futuros docentes según el modelo TPACK. Digital Education Review, 32, 73-84. https://doi.org/10.1344/der.2017.32.73-84

CADE (2016). La educación fuera de la caja. https://www.ipae.pe/wpcontent/uploads/2017/05/revista_cade_05-09.pdf

Carvajal, A. (2018). Teoría y práctica de la sistematización de experiencias. Programa Editorial UNIVALLE.

Diego-Mantecón, J., Blanco, T., Ortiz-Laso, Z., \& Lavicza, Z. (2021). Proyectos STEAM con formato KIKS para el desarrollo de competencias clave. Comunicar, 29(66), 33-43. http://dx.doi.org/10.3916/C66-2021-03

Gonzalez-Diaz, R., Acevedo-Duque, Á., Martin-Fiorino, V. \& Cachicatari-Vargas, E. (2022). Cultura investigativa del docente en Latinoamérica en la era digital. Comunicar: Revista Científica de Comunicación y Educación, 30(70), 71-83. https://doi.org/10.3916/C702022-06

Jara O. (2020). Orientaciones teórico prácticas para la sistematización de experiencias. http://biblioteca.udgvirtual.udg.mx/jspui/handle/123456789/3845

Hernández-Palma, H. G., Niebles-Nuñez, W., Pacheco-Ruiz, C. \& Rojas-Martínez, C. (2020). Estrategias tecnológicas en bibliotecas universitarias como centros de recursos para la investigación y el aprendizaje en la región Caribe de Colombia. Formación universitaria, 13(6), 51-60. http://dx.doi.org/10.4067/S0718-50062020000600051

Llamas-Salguero, F. \& Gómez, E. M. (2018). Formación inicial de docentes en educación básica para la generación de conocimiento con las Tecnologías de la Información y la Comunicación. Revista Complutense de Educación, 29(2), 577. https://doi.org/10.5209/RCED.53520

Mateus, J. C., Andrada, P., González-Cabrera, C., Ugalde, C., \& Novomisky, S. (2022). Perspectivas docentes para una agenda crítica en educación mediática post COVID-19. Estudio comparativo en Latinoamérica. Comunicar: Revista Científica de Comunicación y Educación, 30(70), 9-19. https://www.revistacomunicar.com/pdf/comunicar70.pdf

Ministerio de Educación del Perú (2020) Protocolo de orientaciones pedagógicas para el acompañamiento del aprendizaje durante el período de adaptación de estudiantes del nivel inicial que se trasladan a una institución educativa pública.

Ministerio de Educación del Perú (2021) Orientaciones pedagógicas para prevenir la interrupción de los estudios en el nivel de inicial. https://hdl.handle.net/20.500.12799/7611

Ministerio de Educación del Perú (2017). Currículo Nacional de Educación Básica http://www.minedu.gob.pe/curriculo/pdf/curriculo-nacional-de-la-educacion-basica.pdf 
Superintendencia Nacional de Educación Superior Universitaria (2020). Resolución del Consejo Directivo $N^{o}$ 039-2020-SUNEDU-CD. https://www.sunedu.gob.pe/resolucion-delconsejo-directivo-n-039-2020-sunedu-cd/

Ruiz, G. V. \& Vásquez, M. I. (2019). Estrategias didácticas y atención dispersa en niños de 4 a 5 años. Propuesta. Guía para docentes. Guayaquil: Universidad de Guayaquil. Facultad de Filosofía, letras y ciencias de la educación.

Schwartzman, G., Roni, C., Berk, M., Delorenzi, E., Sánchez, M. \& Eder, M. L. (2021). Evaluación remota de aprendizajes en la universidad: Decisiones docentes para encarar un nuevo desafío. Revista Iberoamericana De Educación a Distancia, 24(2), 67-85. http://dx.doi.org/10.5944/ried.24.2.29078

Trilla, A. (2020). Un mundo, una salud: la epidemia por el nuevo coronavirus COVID-19. Medicina clínica, 154(5), 175. http://dx.doi.org/10.1016/j.medcli.2020.02.002

Unday, D. E. \& Valero, J. A. G. (2017). Sistematización de experiencias como método de investigación. Gaceta Médica Espirituana, 19(2), 10-16. http://scielo.sld.cu/scielo.php?script=sci_arttext\&pid=S1608-89212017000200003

Valencia, W. (2020). Mirada a las prácticas educativas y pedagógicas en tiempos de pandemia. Una reflexión desde el Colectivo de Prácticas de la Facultad de Ciencias de la Educación $y$ de la Red de Prácticas Pedagógicas Investigativas -REDPPI.

Vialart, M. N. (2020). Estrategias didácticas para la virtualización del proceso enseñanza aprendizaje en tiempos de COVID-19. Revista Cubana de Educación Médica Superior, 34(3). http://www.ems.sld.cu/index.php/ems/article/view/2594

Vicentini (2020). La educación superior en tiempos de Covid-19, aportes de la segunda reunión del diálogo virtual con Rectores de Universidades líderes de América Latina https://publications.iadb.org/es/publications/spanish/document/La-educacion-superioren-tiempos-de-COVID-19-Aportes-de-la-Segunda-Reunion-del-Di\%C3\%A1logoVirtual-con-Rectores-de-Universidades-Lideres-de-America-Latina.pdf 